\title{
FDA Regulation of Clinical High Intensity Focused Ultrasound (HIFU) Devices
}

\author{
Gerald R. Harris, Fellow, IEEE
}

\begin{abstract}
In the U. S., medical devices are regulated under the authority of the 1976 Medical Device Amendments to the Food, Drug, and Cosmetic Act, with the Food and Drug Administration's Center for Devices and Radiological Health having primary responsibility. The Act defines several regulatory paths to market depending on the complexity of the device and indications for use. For most high intensity focused ultrasound (HIFU) devices the premarket submissions include both pre-clinical and clinical data. Pre-clinical testing generally comprises ultrasound power measurements and field characterization, in vitro and in vivo temperature measurements, thermal computational modeling, and demonstrating the accuracy for targeting the region of interest and monitoring treatment progress. Protocols for clinical trials are developed by the device sponsor in conjunction with FDA medical and scientific staff. Currently there are no recognized guidance or standards documents for HIFU testing that could be used in the regulatory review process, but such work is underway within the International Electrotechnical Commission.
\end{abstract}

\section{INTRODUCTION}

$\mathrm{A}$ MONG the responsibilities of the U.S. Food and Drug Administration (FDA) is assuring the safety, effectiveness, and truthful labeling of medical devices for human use. Before a medical device can be marketed legally in the U.S., the sponsor desiring to sell the device must seek permission from the FDA. The formal process is defined in the Medical Device Amendments to the U.S. Food, Drug, and Cosmetic Act, which were enacted on May 28, 1976. Under these Amendments, generic device types on the market prior to this date were identified and assigned to one of three regulatory classes based on the level of control necessary to assure the safety and effectiveness of the device. Class I is for the lowest risk devices (e.g., manual stethoscope). These devices are subject to the least regulatory control and in most cases minimal interaction with the FDA is required. However, in a few cases the device sponsor, prior to marketing, must demonstrate substantial equivalence to a preamendments or subsequently cleared device in terms of safety and effectiveness. Class II devices are ones that are deemed a greater risk than Class I devices but are similar to existing devices (e.g., diagnostic ultrasound). For all Class II devices substantial equivalence as just described must be demonstrated. The regulatory

Manuscript received April 7, 2009; revised June 17, 2009.

G.R. Harris is with the Food and Drug Administration, Center for Devices and Radiological Health, Silver Spring, MD 20992, USA (phone: 301-796-2508; fax: 301-796-9934; e-mail: gerald.harris@fda.hhs.gov). submission for a Class II device is termed a "510(k)" (named for a section of the Amendments) premarket notification. Class III is for high risk devices or for devices using new technology in which there is a new intended use or a new type of safety or effectiveness question (e.g., left ventricular assist device). A Premarket Approval (PMA) application is required for these devices. Human clinical trials normally are conducted as part of the regulatory review.

Devices that entered the market after the Amendments were enacted were automatically placed in the class having the most exacting controls (Class III) unless they could be shown to be substantially equivalent to a preamendments device or legally marketed product. For detailed information on device classification the reader may consult www.fda.gov/cdrh/devadvice/313.html .

One medical device application seeing increased clinical research and regulatory activity is high intensity focused ultrasound (HIFU), a technology that is showing promise for localized thermal destruction of a targeted tissue volume with minimal damage to the surrounding region [1]. Current or potential indications for use include treatment of tumors in the prostate, breast, liver, kidney, and brain, debulking uterine fibroids, cardiac ablation during surgery, and stemming blood flow via acoustic cauterization. The primary mechanism in lesion formation is thermal absorption of the HIFU energy. However, cavitation can play a role, and studies are being conducted of tissue destruction via predominately mechanical means using high intensity pulses [2]. The thermally ablated lesion size depends on the focusing properties of the HIFU transducer or transducer array, the local temperature reached, and the exposure time. Both magnetic resonance (MR) and ultrasound imaging have been used for targeting the tissue to be treated. To date MR imaging has achieved greater success in treatment monitoring, but research using ultrasound imaging is ongoing [3].

Section II of this paper outlines the premarket processing of HIFU device submissions within the FDA and describes the regulatory paths to market that have been taken by HIFU devices under both Class II and Class III. In Section III preclinical testing procedures are discussed, and clinical studies are addressed briefly. Section IV addresses current activities in standards development for HIFU. This material is intended to offer some general highlights of the regulatory review process for HIFU devices in the U.S., but it is by no means complete; nor is every relevant topic identified. 
Omitted topics include biocompatibility, software, and labeling. For more comprehensive information, please consult www.fda.gov/cdrh/devadvice/.

\section{Processing HIFU PREMARKET SUBMissions}

Medical device reviews are carried out in the FDA's Center for Devices and Radiological Health (CDRH). The component of CDRH responsible for premarket reviews is the Office of Device Evaluation (ODE). This Office coordinates reviews and renders decisions regarding premarket submissions, authorizes the conduct of clinical trials, coordinates classification of devices, considers reclassification petitions, and interacts with outside advisory panels. ODE calls upon other CDRH Offices for assistance in the reviews, including the Office of Science and Engineering Laboratories (OSEL). OSEL is the laboratory arm of CDRH, and in addition to supporting ODE with technical consulting reviews, its duties include performing product testing in support of premarket and postmarket reviews, developing and evaluating test methods for consensus standards, and coordinating CDRH activities regarding standards development and recognition.

ODE has processed HIFU device submissions under both Class II and Class III, depending on the complexity of the device, desired indications for use, and existence of a substantially equivalent preamendments or cleared device. Class III is generally appropriate for HIFU when used to treat tissues at depth (i.e., greater than a few centimeters below an organ surface), because new types of safety and effectiveness questions likely are raised. For example, a large volume of non-targeted tissue can be exposed both in front of and behind the focal region, such as bowel, bladder, abdominal wall, nerve, or bone. Therefore, methods proposed for targeting accuracy, treatment monitoring, and thermal dosimetry are critical to a safe and effective outcome and need to be evaluated carefully. Also, there are potential risks that are unique to ultrasound compared to other thermal ablation technologies, such as bone absorption, reflection effects, and cavitation.

One application of HIFU has been considered Class II because of its similarity to ablation devices that treat tissue within a few centimeters of the energy source, such as RF and microwave ablation devices. These HIFU devices are indicated for ablation of soft tissue near an organ surface such as the heart during open or laparoscopic surgery. The treatment volume extends from the focal depth of $1-2 \mathrm{~cm}$ back to the surface of the treated organ. Image guidance is not critical because there is no non-targeted region between the transducer and the focus. However, concern about damage to non-targeted tissue beyond focus should be addressed.

\section{Pre-Clinical And Clinical Testing}

Whether Class II or Class III, the submission of preclinical (i.e., bench, in vitro, or in vivo animal) testing is a necessary part of the process to establish that the device is capable of operating in a safe and effective manner. Unlike other medical applications of ultrasound such as physiotherapy, diagnostic imaging, and extracorporeal shock wave lithotripsy, there are no recognized consensus standards or guidance documents for HIFU. Thus, standardized methods currently are unavailable for measuring the acoustic output and beam/focusing characteristics, and for relating these measurements to the distribution of temperature in tissue. In the absence of wellcharacterized standard techniques to obtain this information, the FDA's regulatory evaluations of the pre-clinical testing results are performed on a case-by-case basis. However, the pre-clinical testing data submitted by device sponsors usually include the following elements:

1. Verification of the basic system/transducer design and performance by measuring free-field ultrasonic power output and focusing characteristics

To verify that the transducer output and efficiency are adequate, ultrasonic power typically is measured using a radiation force balance [4], although other techniques based on pyroelectricity and calorimetry are being explored [5]. Beam scanning with a piezoelectric hydrophone is the traditional means for obtaining pressure or intensity maps to determine the focal location and dimensions, but for HIFU the field distribution usually is measured at low output levels to avoid hydrophone damage. Other field mapping measures including robust hydrophone designs, point reflectors, and acoustic streaming are being researched to avoid this damage problem [5]-[7]. Acousto-optic visualization via schlieren imaging also can be useful for finding the focusing characteristics [6].

\section{Demonstration that the device can produce predictable} thermal lesions via both measurements of temperature distributions in vitro and computational modeling

Experimental mapping of the temperature distribution can be accomplished by using non-perturbing thermal sensors embedded in tissue mimicking materials (TMMs) or ex vivo tissue samples, although care should be taken in interpreting the results due to viscous heating and cavitation artifacts [5], [8]. If the TMM is transparent, then ablation lesion locations and dimensions can be evaluated visually, although such a TMM is single-use [9], [10]. One formulation of a reusable TMM has been described in which the following acoustic and thermal properties were characterized: acoustic attenuation, sound speed, nonlinearity parameter $\mathrm{B} / \mathrm{A}$, acoustic backscatter, and thermal conductivity and diffusivity [11]. However, in this TMM the lesions are not visible.

Computational modeling plays an important role in preclinical assessments in that device performance can be predicted under a broader range of conditions than can be investigated experimentally [12], [13]. Intensity plots 
generated using linear or non-linear theoretical propagation models as appropriate are combined with the acoustic properties of tissue to compute heat production rate distributions. Then, using the bio-heat equation and the thermal properties of the relevant tissue(s), temperature profiles and thermal dose contours are found. Examples of use include computing the influence of nearby bone [14], [15] or a large vessel [13], [16], [17] on the heating pattern. Also, modeling techniques that allow rapid computation of the acoustic and thermal fields permit parametric analyses to be performed efficiently, so that the effects of varying the acoustic or thermal properties can be assessed in seconds [18].

3. Demonstration of the safety of non-targeted tissues both proximal and distal to the targeted region through acoustic and thermal measurements and computational modeling

One advantage of HIFU lies in its ability to ablate at depth while sparing tissues not in the focal zone from damage. However, low intensity regions away from the focus may be susceptible to damage at temperatures below those necessary for total ablation [15]. For example, a nerve located near a bony structure beyond the focus could be heated to unacceptable levels due to high absorption in the bone and resultant heat conduction. Another factor to consider is the effect of residual temperature rise should the tissue temperature not return to baseline before a subsequent HIFU exposure is transmitted. Therefore, safety analyses of non-targeted tissues are critical and can be used to prescribe safe zones of treatment as well as temporal operating characteristics for the device.

4. Demonstration of the accuracy of the method for targeting the region of interest and for monitoring the progress or result of treatment, if applicable

To establish that the HIFU device can deliver ultrasonic energy at a clinically appropriate level to precisely-defined tissue locations, the targeting and monitoring capabilities of the system should be described and evaluated. As stated previously, typically either MR or ultrasound imaging is used. Data should be gathered regarding the accuracy of the approach for imaging the targeted region during treatment, for calculating the size and location of the targeted region, and for monitoring the progress or outcome of the treatment, as appropriate.

If thermal dosimetry is employed such as via MR thermometry, then the measurement accuracy should be evaluated. The user interface also should be described, including device feedback to the operator regarding the conduct of the treatment. Further, bubble formation, means for its detection, and possible consequences should be addressed. Sites where bubbles might be expected to occur include the contact surface between the device and patient due to poor coupling, and at or near the focus due to either cavitation or boiling.

After the essential safety and effectiveness of the device have been demonstrated through pre-clinical studies, then clinical studies can be initiated. Clinical data submitted for review should be based on sound scientific principles to demonstrate either substantial equivalence (Class II) or safety and effectiveness (Class III). The clinical study design should include controls and an adequate number of patients, monitoring to assure that the protocol is followed by the investigators, and proper analysis of results. All clinical studies performed in the U.S. in support of a $510(\mathrm{k})$ or PMA must be conducted in accordance with the Investigational Device Exemption (IDE) regulation (21 CFR 812), which requires device sponsors to obtain approval from the FDA of the study before it begins (for significant risk devices), to provide informed consent to each patient, and to conduct proper monitoring of the study. Please consult www.fda.gov/cdrh/devadvice/ide/index.shtml for more information. Clinical data obtained outside of the U.S. may be acceptable if they otherwise meet the criteria for device approval, are applicable to the United States population, medical practice, and requirements for informed consent in conformance with the Declaration of Helsinki, and are from studies performed by clinical investigators of recognized competence.

For further information on clinical studies, please see www.fda.gov/cdrh/devadvice/pma/clinical_studies.html.

\section{Guidance And Consensus Standards DEVELOPMENT}

FDA publishes regulatory guidance documents and participates in national and international standards development for medical devices. This work entails collaborations with standards organizations, the affected industry, other regulatory bodies, and user and other interested groups. Information on FDA's recognition and use of consensus standards, along with a list of recognized standards, can be found at www.fda.gov/cdrh/stdsprog.html

Regarding medical ultrasound devices, FDA has recognized standards developed by the International Electrotechnical Commission (IEC) in the areas of extracorporeal shock wave lithotripsy, physiotherapy, and diagnostics [19]-[24], and a similar process for HIFU device characterization is envisioned. The numerous challenges of this effort have been reported [25], and at present work has begun in IEC Technical Committees TC87, Ultrasonics, and TC62D, Electromedical Equipment. These committees have initiated projects for three HIFU-related documents, one dealing with power measurements, one with field mapping, and one addressing general performance aspects, including mechanical and electrical safety as well as device labeling. These documents have the following designations: (1) IEC 62555 Ed. 1.0, "Ultrasonics - Power measurement - Output 
power measurement for High Intensity Therapeutic Ultrasound (HITU) transducers and systems" (under TC87); (2) IEC 62556 TS Ed. 1.0, "Surgical systems - Specification and measurement of field parameters for High Intensity Therapeutic Ultrasound (HITU) transducers and systems" (under TC87); and (3) IEC 60601-2-XX: "Particular requirements for basic safety and essential performance of high intensity therapeutic ultrasound (HITU) systems" (under TC62D). Note that HITU is being used instead of HIFU to be inclusive of therapeutic devices employing unfocused ultrasound fields. Also, it is planned that proposed IEC 62556 will be a "technical specification" (TS) rather than a full standard, with a standard to follow as experience is gained in this demanding area of ultrasound metrology [5].

The benefits of having consensus standards available are severalfold. Regulatory agencies such as the FDA along with the regulated industry will have recognized means for establishing safety and performance, thus enabling more efficient and scientifically credible regulatory submissions and reviews. Further, the HIFU community will be able to measure and compare device performance including exposure levels more accurately. Finally, standardization should give clinicians more confidence in and patients better access to this emerging medical technology.

\section{REFERENCES}

[1] G. ter Haar and C. Coussios, "High intensity focused ultrasound: Physical principles and devices," Int. J. Hyperthermia, vol. 23, no. 2, pp. 89-104, Mar. 2007.

[2] J.E. Parsons, C.A. Cain, G.D. Abrams, and J.B. Fowlkes, "Pulsed cavitational ultrasound therapy for controlled tissue homogenization," Ultrasound Med. Biol., vol. 32, no. 1, pp. 115-129, Jan. 2006.

[3] I. Rivens, A. Shaw, J. Civale, and H. Morris, "Treatment monitoring and thermometry for therapeutic focused ultrasound," Int. J. Hyperthermia, vol. 23, no. 2, pp. 121-139, Mar. 2007.

[4] S. Maruvada, G.R. Harris, B.A. Herman, and R.L. King, "Acoustic power calibration of high intensity focused ultrasound transducers using a radiation force technique," J. Acoust. Soc. Am., vol. 121, no. 3, pp. 1434-1439, Mar. 2007.

[5] A. Shaw and M. Hodnett, Calibration and measurement issues for therapeutic ultrasound," Ultrasonics, vol. 48, pp. 234-252, Aug. 2008.

[6] G.R. Harris, "Progress in medical ultrasound exposimetry," IEEE Trans. Ultrason. Ferroelec. Freq. Contr., vol. 52, no. 5, pp. 717-736, May 2005.

[7] P. Hariharan, M.R. Myers, R.A. Robinson, S. Maruvada, J. Sliwa, and R.K. Banerjee, "Characterization of high intensity focused ultrasound transducers using acoustic streaming," J. Acoust. Soc. Am., vol. 123, no. 3, pp. 1706-1719, Mar. 2008.

[8] S. Maruvada, Y. Liu, B.A. Herman, and G.R. Harris, "Temperature measurements in tissue-mimicking material during HIFU exposure," in 2008 Proc. 8th Internat. Symp. Therapeutic Ultrasound - to be published.

[9] S. Howard, J. Yuen, P. Wegner and C. Zanelli, "Characterization and FEA simulation for a HIFU phantom material," 2003 Proc. IEEE Ultrasonics Symp., pp. 1270-1273.

[10] C. Lafon, V. Zderic, M.L. Noble, J.C. Yuen, P.J. Kaczkowski, O.A. Sapozhnikov, F. Chavrier, L.A. Crum, and S. Vaezy, "Gel phantom for use in high-intensity focused ultrasound dosimetry," Ultrasound Med. Biol., vol. 31, no. 10, pp. 1383-1389, Oct. 2005.

[11] R.L. King, B.A. Herman, S. Maruvada, K.A. Wear, and G.R. Harris, "Development of a HIFU phantom," 2006 Proc. 8th Internat. Symp. Therapeutic Ultrasound, C.C. Coussios and G. ter Haar. Eds. New York: Amer. Inst. Physics, 2007, pp. 351-356.
[12] C.A. Damianou, K. Hynynen, and X. Fan, "Evaluation of accuracy of a theoretical model for predicting the necrosed tissue volume during focused ultrasound surgery," IEEE Trans. Ultrason. Ferroelec. Freq. Contr., vol. 42, no. 2, pp. 182-187, Mar. 2005.

[13] F.P. Curra, P.D. Mourad, V.A. Khokhlova, R.O. Cleveland, amd L.A. Crum, "Numerical simulations of heating patterns and tissue temperature response due to high-intensity focused ultrasound," IEEE Trans. Ultrason. Ferroelec. Freq. Contr., vol. 47, no. 4, pp. 10771089, July 2005.

[14] M.R. Myers, "Transient temperature rise due to ultrasound absorption at a bone/soft-tissue interface," J. Acoust. Soc. Am., vol. 115, no. 6, pp. 2887-2891, June 2004.

[15] D.M. Nell and M.R. Myers, "Thermal effects generated by highintensity focused ultrasound beams at normal incidence to a bone surface," J. Acoust. Soc. Am., submitted for publication.

[16] J. Huang, R.G. Holt, R.O. Cleveland, and R.A. Roy, "Experimental validation of a tractable numerical model for focused ultrasound heating in flow-through tissue phantoms," J. Acoust. Soc. Am., vol. 116, no. 4, pp. 2451-2458, Oct. 2004.

[17] P. Hariharan, M.R. Myers, and R.K. Banerjee, "HIFU procedures at moderate intensities - effect of large blood vessels," Phys. Med. Biol., vol. 52, no. 12, pp. 3493-3513, Dec. 2007.

[18] J.E. Soneson, "A user-friendly software package for HIFU simulation," 2008 Proc. 8th Internat. Symp. Therapeutic Ultrasound to be published.

[19] Ultrasonics - Pressure pulse lithotripters - Characteristics of fields, IEC 61846 Ed. 1.0, Geneva: Internat. Electrotech. Comm., 1998.

[20] Particular requirements for the safety of equipment for extracorporeally induced lithotripsy, IEC 60601-2-36 Ed. 1.0, Geneva: Internat. Electrotech. Comm., 1997.

[21] Ultrasonics - Physiotherapy systems - Performance requirements and methods of measurement in the frequency range $0.5 \mathrm{MHz}$ to $5 \mathrm{MHz}$, IEC 61689 Ed. 2.0, Geneva: Internat. Electrotech. Comm., 2000.

[22] Particular requirements for the safety of ultrasonic physiotherapy equipment, IEC 60601-2-5 Ed. 2.0, Geneva: Internat. Electrotech. Comm., 2005.

[23] Ultrasonics - Field characterization - Test methods for the determination of thermal and mechanical indices related to medical diagnostic ultrasonic fields, IEC 62359 Ed. 1.0, Geneva: Internat. Electrotech. Comm., 2005.

[24] Particular requirements for the basic safety and essential performance of ultrasonic medical diagnostic and monitoring equipment, IEC 60601-2-37 Ed. 2.0, Geneva: Internat. Electrotech. Comm., 2007.

[25] A. Shaw and G. ter Haar, "Requirements for measurement standards in high intensity focused ultrasound (HIFU) fields," National Physical Laboratory, Teddington, Middlesex, UK, NPL Report DQL AC 015, Feb. 2006. 